\title{
Proteomic analysis of oxidative stress response in human umbilical vein endothelial cells (HUVECs): role of heme oxygenase 1 (HMOX1) in hypoxanthine-induced oxidative stress in HUVECs
}

\author{
Pei Zhu ${ }^{1 \#}$, Tao Qi ${ }^{1 \#}$, Zhan-Sen Huang ${ }^{1}$, Hao Li $^{2}$, Bo Wang ${ }^{1}$, Jia-Xin Feng ${ }^{3}$, Shuai Ma ${ }^{3}$, Heng-Jun Xiao ${ }^{4}$, \\ Yu-Xin Tang ${ }^{5}$, Wei Liu' ${ }^{6}$ Jun Chen ${ }^{1}$ \\ ${ }^{1}$ Department of Infertility and Sexual Medicine, the Third Affiliated Hospital of Sun Yat-sen University, Guangzhou 510630, China; ${ }^{2}$ Department \\ of Urology, the Third Affiliated Hospital of Guangzhou Medical University, Guangzhou 510150, China; ${ }^{3}$ Guangxi University of Chinese Medicine, \\ Nanning 530200, China; ${ }^{4}$ Department of Urology, the Third Affiliated Hospital of Sun Yat-Sen University, Guangzhou 510630, China; ${ }^{5}$ Department \\ of Urology, The Fifth Affiliated Hospital of Sun Yat-sen University, Zhuhai 519000, China; ${ }^{6}$ Guangdong Provincial Key Laboratory of Liver Disease, \\ the Third Affiliated Hospital of Sun Yat-Sen University, Guangzhou 510630, China \\ Contributions: (I) Conception and design: J Chen, W Liu, YX Tang, HJ Xiao; (II) Administrative support: None; (III) Provision of study materials or \\ patients: None; (IV) Collection and assembly of data: H Li, B Wang, P Zhu, JX Feng, S Ma; (V) Data analysis and interpretation: P Zhu, T Qi, ZS \\ Huang; (VI) Manuscript writing: All authors; (VII) Final approval of manuscript: All authors. \\ \#These authors contributed equally to this work. \\ Correspondence to: Dr. Jun Chen. Department of Infertility and Sexual Medicine, The Third Affiliated Hospital of Sun Yat-Sen University, No. 600 \\ Tianhe Road, Guangzhou 510630, China. Email: jchen121121@hotmail.com; Dr. Wei Liu. Guangdong Provincial Key Laboratory of Liver Disease, \\ the Third Affiliated Hospital of Sun Yat-sen University, No. 600 Tianhe Road, Guangzhou 510630, China. Email: lwei6@mail.sysu.edu.cn; Dr. Yu-Xin \\ Tang. Department of Urology, The Fifth Affiliated Hospital of Sun Yat-sen University, Zhuhai 519000, China. Email: tangyx36@mail.sysu.edu.cn.
}

Background: Erectile dysfunction (ED) is a well-known complication of diabetes, affecting up to $75 \%$ of diabetic men. Although the etiology of diabetic ED is multifactorial, endothelial dysfunction is considered to be a pillar of its pathophysiology. Endothelial dysfunction is caused by the harmful effects of high glucose levels and increased oxidative stress on the endothelial cells that comprise the vascular endothelium. The aim of this study was to identify the proteomic changes caused by high glucose-induced oxidative stress and explore the role of heme oxygenase 1 (HMOX1) in it.

Methods: The cellular proteomic response to hypoxanthine-induced oxidative stress in human umbilical vein endothelial cells (HUVECs) was analyzed by isobaric tags for relative and absolute quantitation (iTRAQ) combined with liquid chromatography-tandem mass spectrometry (LC-MS/MS). Differentially expressed proteins (DEPs) were analyzed through Network and Kyoto Encyclopedia of Genes and Genomes (KEGG) pathway analyses. Further validation assays was performed to validate the role of HMOX1.

Results: The results showed that 66 and 76 DEPs were markedly upregulated and downregulated, respectively, for HUVECs oxidative stress. Among these proteins, we verified eight dysregulated genes by quantitative reverse transcription PCR, including nucleolin (NCL), X-ray repair cross-complementing protein 6 (XRCC6), ubiquinol-cytochrome C reductase binding protein (UQCRB), non-POU domain containing octamer binding (NONO), heme oxygenase 1 (HMOX1), nucleobindin 1 (NUCB1), DEK, and chromatin target of prmt1 (CHTOP). Further, using overexpression and genetic knockdown approaches, we found that HMOX1 was critical for the oxidative stress response in HUVECs.

Conclusions: We found that HMOX1 was closely related to the oxidative stress response induced by hypoxanthine. To the best of our knowledge, this study is the first overview of the responses of HUVECs to oxidative stress. The findings will contribute to analyses of the detailed molecular mechanisms involved in the pathogenesis of endothelial dysfunction and related molecular mechanisms in ED patients.

Keywords: Erectile dysfunction (ED); hypoxanthine; human umbilical vein endothelial cells (HUVECs); oxidative stress; isobaric tags for relative and absolute quantitation (iTRAQ); liquid chromatography-tandem mass 
spectrometry (LC-MS/MS); heme oxygenase 1 (HMOX1)

Submitted Nov 23, 2019. Accepted for publication Jan 29, 2020.

doi: $10.21037 /$ tau.2020.03.11

View this article at: http://dx.doi.org/10.21037/tau.2020.03.11

\section{Introduction}

With improvements in the standard of living, the incidence of diabetes is increasing rapidly, and the number of patients diagnosed with diabetes is expected to rise to approximately 300 million worldwide by 2025 (1). Diabetic erectile dysfunction (DED) is a common complication of diabetes, with an incidence of more than $50 \%$ in diabetic men (2). Erectile dysfunction (ED) is a common disease in men. It is defined as periodic or persistent ED of the penis, which cannot achieve or maintain erectile function sufficient to satisfy sexual behavior (3-5). Penile erection is a complex physiological process regulated by the central and peripheral nervous systems, vascular system, and endocrine system (5). It requires the collaboration of multiple factors, including normal vascular endothelial cells (VECs) and the corpus cavernosum; any abnormalities in these processes may lead to ED (6). The vascular endothelium, a simple squamous epithelial layer on the surface of blood vessels, plays a major role in endocrine signaling and has a wide variety of biological functions $(7,8)$, including the process of penile erection (9). Endothelial dysfunction, caused by harmful changes due to high glucose levels and increased oxidative stress in endothelial cells, is critical for the progression of DED (10). In addition to direct damage to endothelial cells, diabetes may hinder the angiogenesis implicated in vascular repair mechanisms, further affecting vasodilation and cavernous blood perfusion, which are essential for normal erectile function.

Hyperglycemia causes various metabolic disorders and promotes endothelial dysfunction and vascular complications (11). Chronic high glucose levels are thought to induce the formation of advanced glycation endproducts (12), as well as the generation of reactive oxygen species (ROS) and reactive nitrogen species (RNS) (13-15). The increased production of ROS and RNS, especially the free radical superoxide anion $\left(\mathrm{O}_{2}^{-}\right)$that reacts with nitric oxide $(\mathrm{NO})$ to form peroxynitrite anion $\left(\mathrm{ONOO}^{-}\right)$, has been shown to interfere with endothelial NO bioavailability, propagate endothelial dysfunction, and impair endothelial and cavernosal smooth muscle reactivity (16).
Hyperglycemia-induced dysregulation of erectile-related signaling pathways in endothelial cells, which mimic the pathological process of DED, provide an effective way to find potential molecular pathologic and therapeutic targets in DED. Thus, the current study aimed to investigate the underlying mechanisms of hyperglycemia-induced endothelial dysfunction using proteomic approaches.

\section{Methods}

\section{Cell culture and oxidative stress treatment}

Human umbilical vein endothelial cells (HUVECs) were cultured in RPMI-1640 supplemented with $50 \mathrm{U} / \mathrm{mL}$ penicillin, $50 \mathrm{U} / \mathrm{mL}$ streptomycin (Invitrogen, Carlsbad, CA, USA), and 10\% fetal bovine serum (FBS; Gibco, Grand Island, NY, USA). The cells were incubated at $37^{\circ} \mathrm{C}$ in a humidified atmosphere containing $5 \% \mathrm{CO}_{2}$. To establish an in vitro model of HUVECs oxidative stress and endothelial dysfunction, HUVECs were treated with different concentrations of hypoxanthine in order to observe their oxidative damage. HUVECs were prepared as a suspension of $1.5 \times 10^{5}$ cells $/ \mathrm{mL}$ and then seeded in $24-$ well plates at $1 \mathrm{~mL}$ per well. When the cells reached $80 \%$ confluence, they were divided into the normal control group and treatment groups. Specifically, as previously described (17), HUVECs were treated with hypoxanthine (dissolved in PBS) at a final concentration of $500,1,000,2,000$, or $5,000 \mathrm{ng} / \mathrm{mL}$. Three wells were used for each treatment group. The cells were further incubated at $37^{\circ} \mathrm{C}$ and $5 \% \mathrm{CO}_{2}$, and then visualized with an inverted microscope to reveal cell morphology.

\section{Sample preparation, protein extraction, digestion, and isobaric tags for relative and absolute quantitation (iTRAQ) labeling}

HUVECs were collected from the normal control and $1,000 \mathrm{ng} / \mathrm{mL}$ hypoxanthine-treated groups. All samples were analyzed in triplicate, and each independent sample was analyzed in three technical replicates. Protease inhibitor was added in the mixture of the lysate, and then 
collected cells for lysis. The lysed cells were sonicated and centrifuged at 14,000 $\times \mathrm{g}$ for $40 \mathrm{~min}$. Protein content in the supernatant was then quantified using the bicinchoninic acid (BCA) Protein Assay Kit (Bio-Rad, Hercules, CA, USA). Then, $200 \mu \mathrm{g}$ of protein was digested with $4 \mu \mathrm{g}$ trypsin (Promega, Madison, WI, USA) at $37^{\circ} \mathrm{C}$ overnight. According to the protocol of the iTRAQ kit (8plex, Applied Biosystems, Foster City, CA, USA), $100 \mu \mathrm{g}$ of the resulting peptide mixture from each sample was labeled as follows: three normal control samples were labeled with iTRAQ 113,114 , or 115 , and three treated samples were labeled with iTRAQ 117,118 , or 119 . Then, the labeled samples were mixed and dried with a rotary vacuum concentrator.

\section{Fractionation and liquid chromatography-tandem mass spectrometry (LC-MS/MS)}

The labeled peptides were reconstituted with high-pH reverse-phase (RP) liquid phase $\left(20 \mathrm{mM} \mathrm{HCOONH}_{4}\right.$, $\mathrm{pH} 10)$ and mixed. According to the peak type and time, 24 components were collected in Eppendorf tubes from a linear gradient, acidified with $50 \%$ trifluoroacetic acid, vacuum-dried, and analyzed by two-dimensional liquid chromatography-mass spectrometry (LC-MS). The vacuum-dried sample was resuspended in $20 \mu \mathrm{L}$ highperformance liquid chromatography (HPLC) Buffer A (0.1\% formic acid, $2 \%$ acetonitrile), injected into a ZORBAX 300-C18 RP column $(5 \mu \mathrm{m}, 300 \AA$ A $0.1 \mathrm{~mm} \times$ $150 \mathrm{~mm})$, and then equilibrated with Buffer B $(0.125 \%$ formic acid, 95\% ACN). The sample was eluted with a concentration gradient of acetonitrile $(5-35 \%$ in $0.1 \%$ formic acid) in $90-\mu \mathrm{m}$ volumes with a flow rate of $0.3 \mu \mathrm{L} / \mathrm{min}$. The eluted sample was then analyzed by Q-Orbitrap System first-order mass spectrometry (MS) and tandem mass spectrometry (MS/MS) (Thermo Fisher Scientific, Waltham, MA, USA).

\section{Bioinformatics analysis}

ProteinPilot Software 5.0 (ABSciex, Redwood City, CA, USA) was used to identify and quantify differentially expressed proteins (DEPs) from the LC-MS/MS data. To further understand the impact of DEPs on endothelial cells and to investigate relationships between the DEPs, gene ontology (GO) enrichment, Kyoto Encyclopedia of Genes and Genomes (KEGG) pathway enrichment, and protein-protein interaction (PPI) analyses were performed. GO enrichment was performed for biological process, molecular function, and cellular component, and was applied based on Fisher's exact test considering the whole quantified protein annotations as the background dataset. The Benjamini-Hochberg correction for multiple testing was further applied to adjust the derived $\mathrm{P}$ values. In our iTRAQ proteomic analysis, the screening was based on the following criteria: $\mathrm{P}<0.05$ and fold change $(\mathrm{FC})$ ratio $\geq 1.3$ or $\leq 0.76$. GO terms with $\mathrm{P}<0.05$ and false discovery rate $(\mathrm{FDR})<0.05$ were considered significantly enriched. KEGG pathway enrichment was performed using the clusterProfiler package in $\mathrm{R}$ software (version 3.6.0) based on the KEGG pathway database (http://www.kegg.jp/ $\mathrm{kegg} /$ pathway.html). PPI network analysis was performed according to the STRING database (http://string-db.org/). Then, the results were imported into CytoScape software for visualization.

\section{Quantitative reverse transcription PCR (RT-qPCR)}

HUVECs were harvested using trypsin and lysed with TRIzol Reagent (Invitrogen, Carlsbad, CA, USA). Total RNA was extracted and purified using the RNeasy Mini Kit (Qiagen, Hilden, Germany) according to the manufacturer's protocol and eluted with nuclease-free water. First-strand cDNA was synthesized using PrimeScript RT Master Mix (Thermo Fisher Scientific). The primers used for real-time PCR are listed in Table 1.

\section{Immunofluorescence}

HUVECs were plated in 4-well, 35-mm dishes (Greiner Bio-One, Kremsmünster, Austria) at a density of 1,000 cells/well and grown for $48 \mathrm{~h}$. Then, the cells were fixed with $4 \%$ paraformaldehyde for $20 \mathrm{~min}$ and permeabilized in PBS supplemented with $0.5 \%$ Triton $\mathrm{X}-100$. After blocking, the indicated antibodies were added to the cells and incubated for $2 \mathrm{~h}$. The cells were washed in PBS, incubated with tetramethylrhodamine (TRITC)or fluorescein isothiocyanate (FITC)-labeled secondary antibodies (Pierce Biotechnology, Waltham, MA, USA) for $1 \mathrm{~h}$ at room temperature, and stained with 4,6-diamidino-2phenylindole (DAPI). The cells were mounted with glycerol and observed using a Nikon A1 laser scanning confocal microscope (Tokyo, Japan).

\section{Transfection and Western blotting}

HUVECs were harvested using trypsin, lysed with RIPA 
Table 1 Primers used for RT-qPCR

\begin{tabular}{|c|c|}
\hline Gene & Primer sequence \\
\hline NCL & Reverse: 5'-AAАCAGTCCATTTAАTCTCTGACCTCACG-3' \\
\hline \multirow[t]{2}{*}{ XRCC6 } & Forward: 5'-AAGAATGTCTCCCCTTATTTTGTGG-3' \\
\hline & Reverse: 5'-TCTCGAAACTGTCGCTCCTGTATGT-3' \\
\hline UQCRB & Forward: 5'-ATGTGAATTCATGGCTGGTAAGCAGGCC-3' \\
\hline \multirow[t]{2}{*}{ NONO } & Forward: 5'-AAAGCAGGCGAAGTTTTCATTC-3' \\
\hline & Reverse: 5'-ATCCCGCTGACTGTTCCCT-3' \\
\hline \multirow[t]{2}{*}{ HMOX1 } & Forward: 5'-CACTTCGTCAGAGGCCTGCTA-3' \\
\hline & Reverse: 5'-GTCTGGGATGAGCTAGTGCTGAT-3' \\
\hline NUCB1 & Forward: 5'-СTGCTCAAGGCCAAGATGGA-3' \\
\hline \multirow[t]{2}{*}{ СНTOP } & Forward: 5'-AGAGAGGCTTGCCCAGAGG-3' \\
\hline & Reverse: 5'-CCGACCTATCATACCCCGAC-3' \\
\hline \multirow[t]{2}{*}{$\beta$-actin } & Forward: 5'-GTTGACATCCGTAAAGACC-3' \\
\hline & Reverse: 5'-TAGGAGCCAGGGCAGTAATC-3' \\
\hline
\end{tabular}

RT-qPCR, reverse transcription PCR.

buffer, and the supernatants were collected by centrifugation. The protein content in the RIPA lysates is quantified, and the concentration was determined using the BCA Protein Assay reagent kit. Proteins were fractionated using SDSPAGE and transferred to polyvinylidene fluoride (PVDF) membranes; the membranes were blocked for $1 \mathrm{~h}$ in $5 \%$ skim milk before being incubated with antibodies against heme oxygenase 1 (HMOX1), p-AKT1, and AKT1 (Abcam, UK) at $4{ }^{\circ} \mathrm{C}$ overnight. The membranes were then washed three times in TBST (Tris-buffered saline, 0.1\% Tween 20) for 10 min each. Then, each membrane was incubated with antirabbit IgG H\&L for $1 \mathrm{~h}$ at room temperature. Finally, each membrane was washed again with TBST and bound protein was visualized using an enhanced chemiluminescence (ECL) Western blotting detection kit (GE Healthcare, Chicago, IL, USA); signals were observed using GeneGnome (Syngene, Bangalore, India). For HMOX1 gene silencing and overexpression, the cells were transfected with plasmids designed by OBiO Technology (Shanghai, China).

\section{Cell counting assay}

The effect of shRNA-HMOX1 on HUVEC proliferation was examined using the Cell Counting Kit-8 (CCK-8) assay (Dojindo, Kumamoto, Japan). Briefly, the cells were incubated in $100 \mu \mathrm{L}$ medium in 96-well plates at 2,000 cells/well. Then, $10 \mu \mathrm{L}$ CCK- 8 reagent was added to each well and incubated for $2 \mathrm{~h}$ at $37^{\circ} \mathrm{C}$, and a microplate reader was used to determine the absorbance at $450 \mathrm{~nm}$.

\section{Statistical analysis}

Data are reported as the means \pm SD, and statistical analyses were performed using Graphpad Prism version 7.0 (GraphPad Software, San Diego, California, USA). Comparisons were performed by one-way ANOVA or independent Student's $t$-tests. A value of $\mathrm{P}<0.05$ was considered statistically significant.

\section{Results}

\section{Oxidative stress model of cultured HUVECs}

Endothelial dysfunction is caused by the harmful effects of increased glucose and oxidative stress on endothelial cells (2). To further investigate the underlying mechanism of oxidative stress-induced endothelial dysfunction in DED patients, we stimulated HUVECs with the ROS generator hypoxanthine 

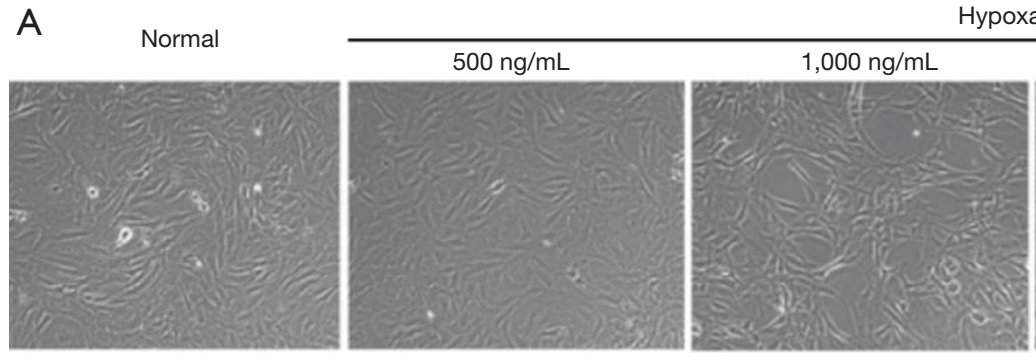

Hypoxanthine
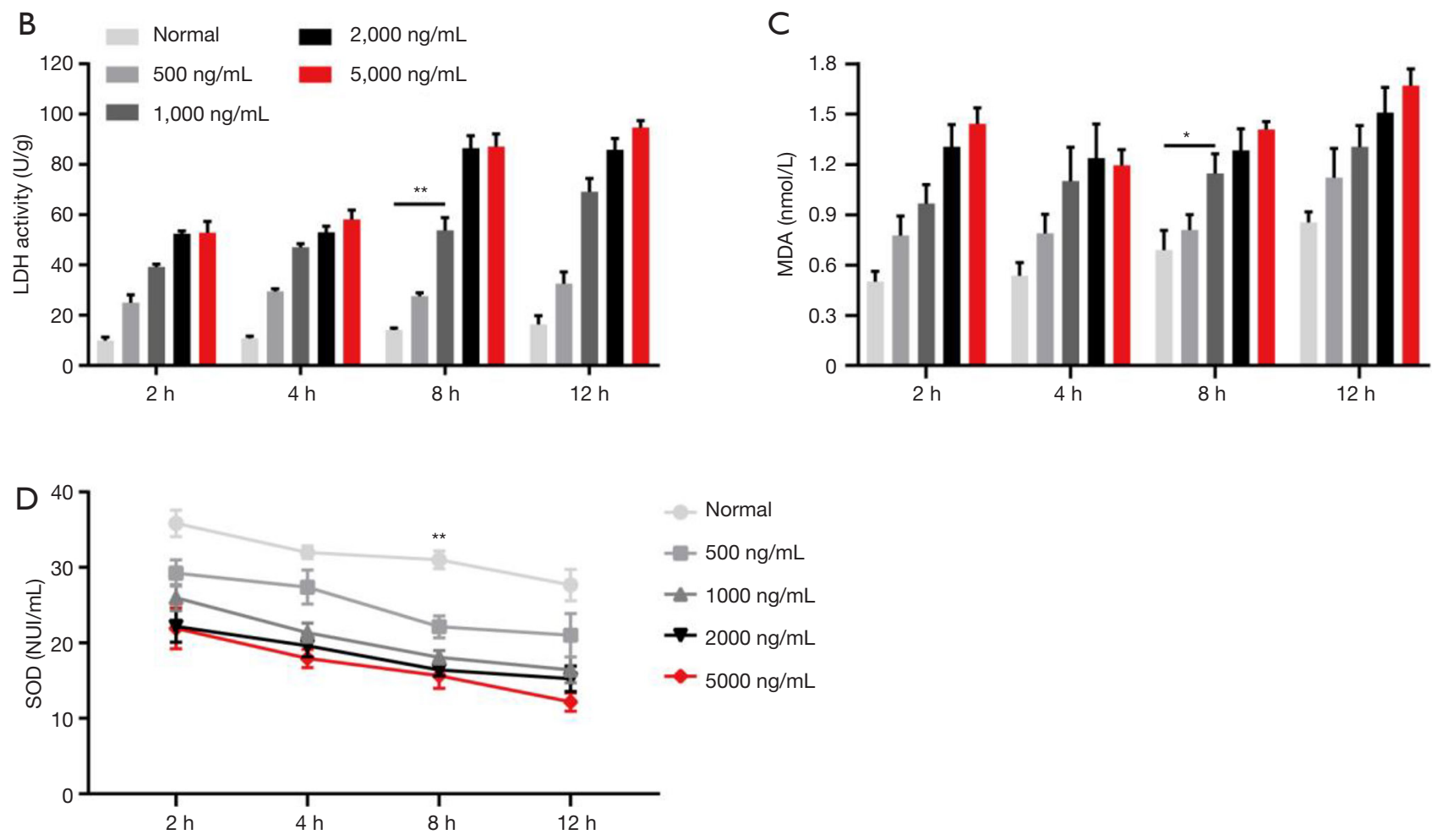

Figure 1 Hypoxanthine treatment induces oxidative stress response in human umbilical vein endothelial cells (HUVECs). HUVECs were treated without or with hypoxanthine at 500, 1,000, 2,000, or 5,000 ng/mL for 2, 4, 8, or $12 \mathrm{~h}$. (A) Representative images of HUVECs under each treatment. Scale bar, $20 \mu \mathrm{m}$; (B,C,D) the levels of lactate dehydrogenase (LDH) (B), malondialdehyde (MDA) (C), and superoxide dismutase (SOD) (D) were measured. Data represent the means $\pm \mathrm{SD}$ from three independent experiments in triplicate. * $\mathrm{P}<0.051,000$ ng/mL versus the normal group; ${ }^{* *}, \mathrm{P}<0.011,000 \mathrm{ng} / \mathrm{mL}$ versus the normal group by one-way ANOVA.

to mimic an oxidative stress model $(3,18,19)$. Four different concentrations of hypoxanthine were added to HUVECs for 2, 4, 8, and $12 \mathrm{~h}$. Lactate dehydrogenase (LDH) activity, malondialdehyde (MDA) levels, and superoxide dismutase (SOD) activity were measured by ELISA. As shown in Figure 1A, cultured HUVECs in the control group formed a flattened monolayer and displayed typical cobblestone-like morphology at confluence. Upon hypoxanthine treatment, the HUVECs were rearranged and interconnected sparsely; damaged and floating cells were apparent, and the cell density decreased significantly in a hypoxanthine concentration-dependent manner (Figure 1A). Furthermore, the levels of LDH (Figure 1B) and MDA (Figure 1C) were increased, while SOD activity was decreased (Figure 1D) by hypoxanthine treatment in a time- and concentrationdependent manner. For subsequent experiments, we chose $1,000 \mathrm{ng} / \mathrm{mL}$ hypoxanthine treatment for HUVECs; at this concentration, no visible morphological damage occurred, but significant changes were observed in LDH, MDA, and SOD levels. 

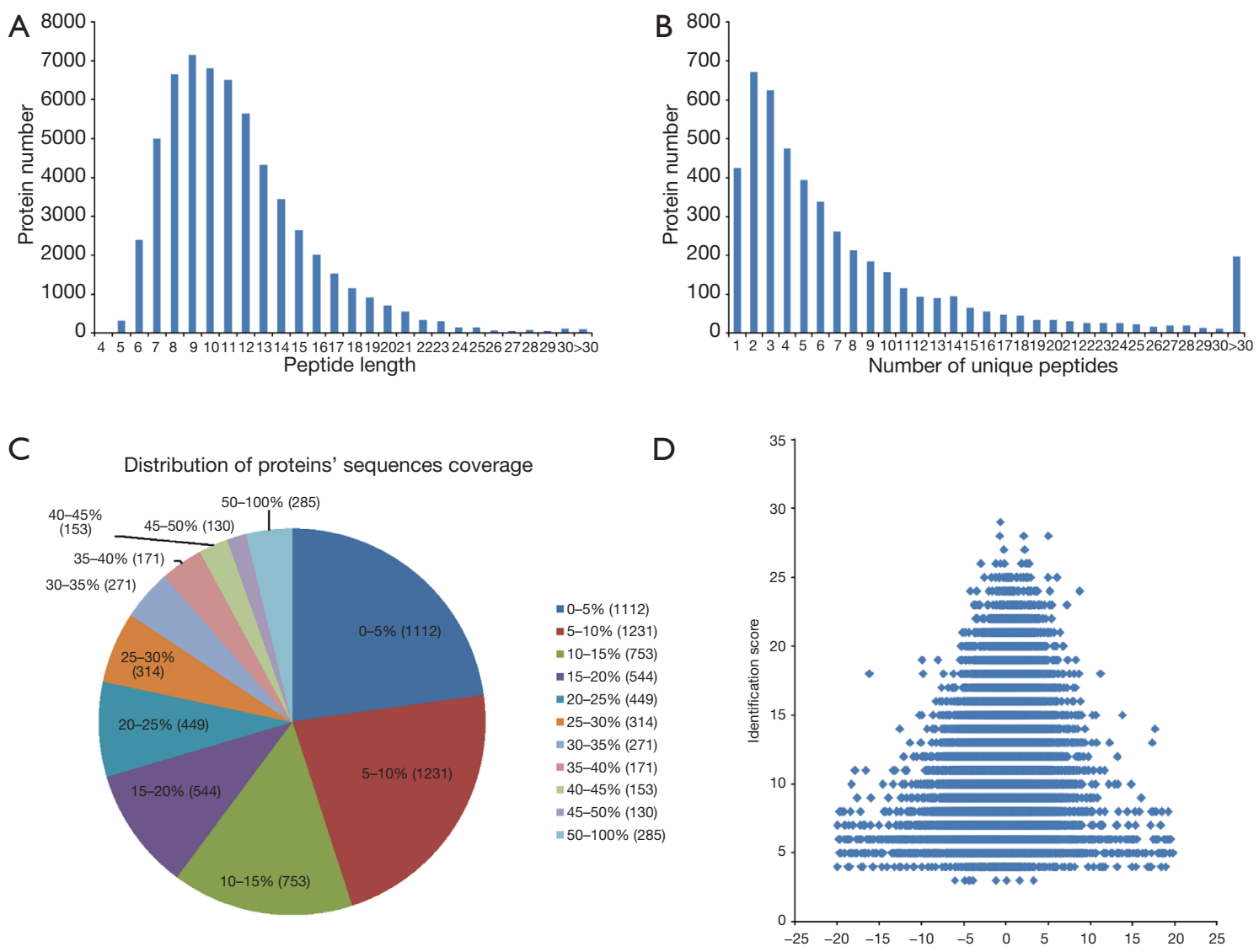

Figure 2 Results of isobaric tags for relative and absolute quantitation analysis. (A) Distribution of peptide lengths; (B) unique peptides in the detected proteins; (C) coverage of proteins identified by liquid chromatography-tandem mass spectrometry (LC-MS/MS); (D) error distribution of spectrogram quality matching.

\section{General information on iTRAQ analysis}

Cell samples were collected and prepared for iTRAQ analysis. iTRAQ-coupled LC-MS/MS analysis identified 40,868 peptides and 4,769 proteins (Figure 2). Most peptides were 6-21 amino acids in length, with the majority being 9 amino acids in length (Figure 2A). Among the 4,796 identified proteins, most contained fewer than ten peptides, and the protein amount decreased as the number of matched peptides increased (Figure 2B). Next, the distribution of protein sequence coverage was analyzed (Figure 2C). The majority of the identified proteins showed good peptide coverage; $50.8 \%$ of the identified proteins had more than $10 \%$ peptide coverage, and $23.6 \%$ had more than $20 \%$ coverage. The matching error distribution of the peptides is shown in Figure 2D. These results suggested that the protein isolation and identification were successful, and the data were subjected to further analysis.

\section{Identification of DEPs in oxidatively stressed HUVECs}

Three groups of normal control samples were labeled with iTRAQ 113, 114, and 115, respectively, and three groups of oxidized samples were labeled with iTRAQ 117, 118, and 119 , respectively. We used principal component analysis (PCA) to test the correlation between the values of the three biological replicates, and found that a proportion of the analyzed proteins was responsible for hypoxanthineinduced oxidative stress in HUVECs (Figure $3 A$ ). In our iTRAQ proteomic analysis, the screening was based on the following criteria: $\mathrm{P}<0.05$ and $\mathrm{FC}$ ratio $\geq 1.3$ or $\leq 0.76$. Among the DEPs, 66 proteins were significantly upregulated and 76 were significantly downregulated (Figure 3B). The top 20 DEPs are shown in Table 2. The 

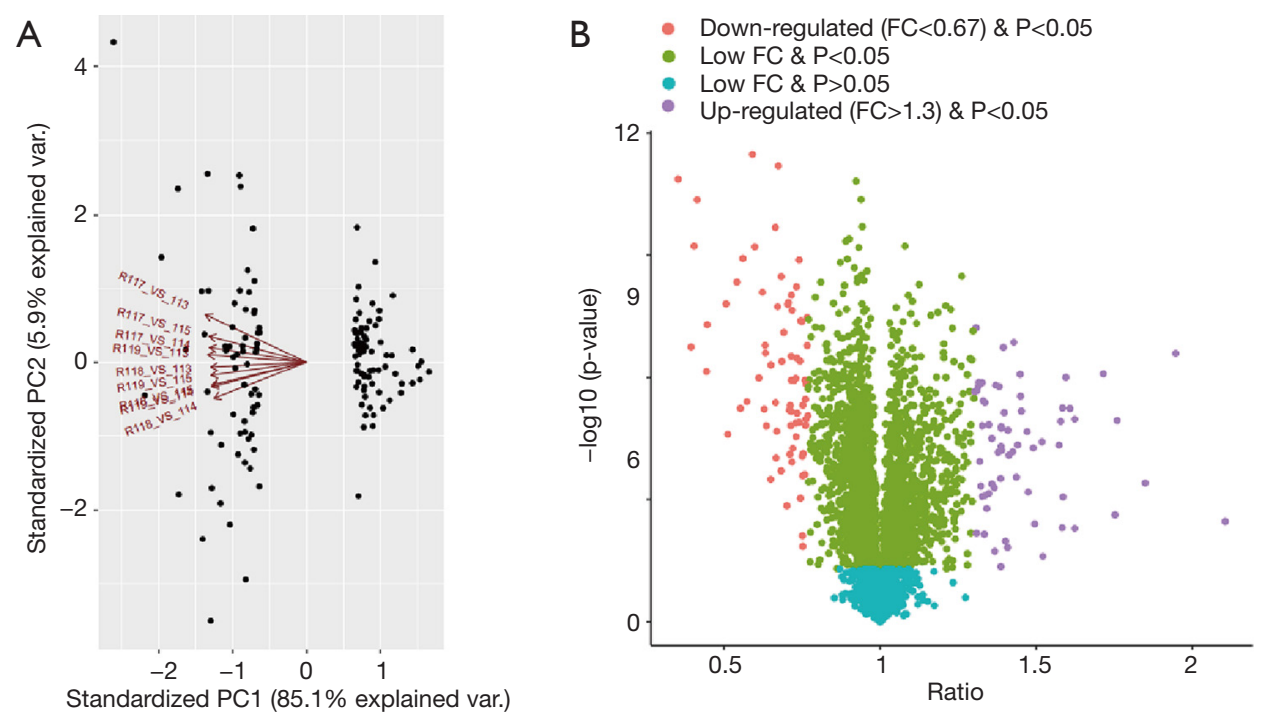

Figure 3 Analysis of differentially expressed proteins (DEPs) between hypoxanthine-treated and untreated human umbilical vein endothelial cells (HUVECs). (A) Principal component analysis plot showing the corrected correlation values of the three biological replicates; (B) volcano plot of DEPs. FC, fold change.

Table 2 Top 20 differentially expressed proteins in HUVECs under hypoxanthine-induced oxidative stress

\begin{tabular}{|c|c|c|}
\hline Accession & Gene symbol & FC ratio (model/control) \\
\hline \multicolumn{3}{|l|}{ Upregulated } \\
\hline sp|Q86WR0 & $C C D 25$ & 2.104782303 \\
\hline sp|P27695 & APEX1 & 1.946251088 \\
\hline sp|O60784 & TOM1 & 1.848857416 \\
\hline sp|Q9Y399 & RT02 & 1.758804427 \\
\hline $\operatorname{tr||3L504~}$ & I3L504 & 1.752057433 \\
\hline sp|Q16543 & CDC37 & 1.714400662 \\
\hline sp|Q96K37 & S35E1 & 1.623314752 \\
\hline sp|Q5SNV9 & CA167 & 1.623209715 \\
\hline sp|P04818 & TYSY & 1.607742018 \\
\hline sp|P53999 & TCP4 & 1.594754683 \\
\hline sp|Q96QC0 & PP1RA & 1.586030126 \\
\hline sp|Q06830 & PRDX1 & 1.584336493 \\
\hline sp|Q16706 & $M A 2 A 1$ & 1.583226217 \\
\hline sp|Q8IU81 & $\mid 2 B P 1$ & 1.578175558 \\
\hline sp|Q9UH65 & SWP70 & 1.573015438 \\
\hline sp|Q9BVG9 & PTSS2 & 1.521094534 \\
\hline sp|O15511 & ARPC5 & 1.518378933 \\
\hline $\mathrm{sp} \mid \mathrm{O} 15446$ & RPA34 & 1.495074259 \\
\hline sp|P62979 & $R S 27 A$ & 1.490537537 \\
\hline sp|Q4VCS5 & AMOT & 1.474095782 \\
\hline
\end{tabular}

Table 2 (Continued)
Table 2 (Continued)

\begin{tabular}{|c|c|c|}
\hline Accession & Gene symbol & FC ratio (model/control) \\
\hline \multicolumn{3}{|l|}{ Downregulated } \\
\hline sp|P49588 & SYAC & 0.353742354 \\
\hline sp|P62805 & H4 & 0.395187226 \\
\hline sp|Q9BV57 & MTND & 0.404758182 \\
\hline sp|P63261 & ACTG & 0.414486246 \\
\hline sp|P63244 & RACK1 & 0.444610609 \\
\hline sp|P35527 & $\mathrm{K} 1 \mathrm{C9}$ & 0.446953376 \\
\hline sp|P09132 & SRP19 & 0.507089072 \\
\hline sp|Q9NV92 & NFIP2 & 0.512578726 \\
\hline sp|Q9Y3Y2 & CHTOP & 0.5417456 \\
\hline sp|P62888 & $R L 30$ & 0.552873929 \\
\hline sp|P09601 & HMOX1 & 0.560592135 \\
\hline sp|Q9BW92 & SYTM & 0.573749512 \\
\hline $\operatorname{tr|A0A087WWE2~}$ & A0A087WWE2 & 0.591824353 \\
\hline sp|Q92896 & GSLG1 & 0.599185434 \\
\hline sp|P35914 & HMGCL & 0.612094243 \\
\hline sp|P04264 & $\mathrm{K} 2 \mathrm{C} 1$ & 0.623481936 \\
\hline sp|P04406 & G3P & 0.629085359 \\
\hline sp|Q9BY77 & PDIP3 & 0.631970955 \\
\hline sp|094925 & GLSK & 0.633027938 \\
\hline sp|Q02818 & NUCB1 & 0.63618553 \\
\hline
\end{tabular}



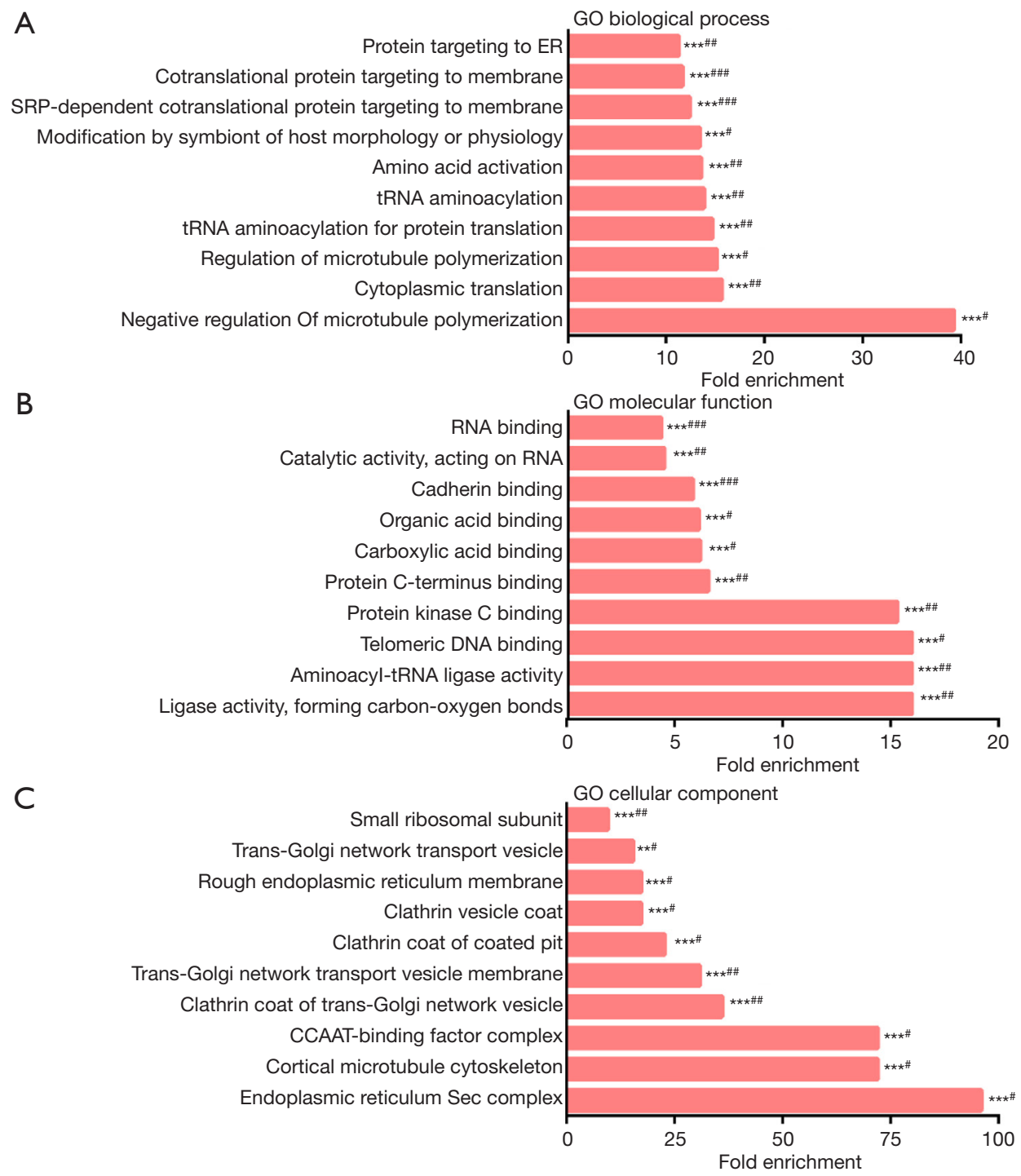

Figure 4 Gene ontology (GO) term enrichment of the identified dysregulated proteins. ${ }^{* *}, \mathrm{P}<0.01$; ${ }^{* * *}, \mathrm{P}<0.001$; ${ }^{*}$, false discovery rate (FDR) $<0.05 ;$;, FDR $<0.01 ;$;, FDR $<0.001$.

detail information of the DEPs is shown as a heatmap in Figure S1.

\section{Functional characterization of the DEPs}

To study the biological functions of the 142 DEPs, GO enrichment was performed. GO terms with $\mathrm{P}<0.05$ and FDR $<0.05$ were considered significantly enriched. Negative regulation of microtubule polymerization, cytoplasmic translation, and regulation of microtubule polymerization were the most highly enriched under biological process (Figure $4 A$ ). Ligase activity, formation of carbon-oxygen bonds, aminoacyl-tRNA ligase activity, and telomeric DNA binding were the most highly enriched under molecular function (Figure 4B). Endoplasmic reticulum Sec complex, cortical microtubule cytoskeleton, and CCAAT-binding factor complex were the most highly enriched under cellular component (Figure 4C).

\section{KEGG pathway annotation of DEPs}

In order to further study and screen the pathways related to DEPs, we conducted KEGG pathway analysis. $\mathrm{P}<0.05$ was defined as significant KEGG pathway enrichment. 
Table 3 KEGG pathway analysis of dysregulated proteins in HUVECs under oxidative stress

\begin{tabular}{llll}
\hline Pathway ID & Description & Genes in this pathway (gene ID) & P value \\
\hline hsa03060 & Protein export & $23478,6734,10952,6728$ & $1.71 \times 10^{-8}$ \\
hsa05131 & Shigellosis & $1399,10092,960,4793,71$ & $1.28 \times 10^{-7}$ \\
hsa00970 & Aminoacyl-tRNA biosynthesis & $8565,4677,16,57505,80222$ & $1.41 \times 10^{-7}$ \\
hsa05100 & Bacterial invasion of epithelial cells & $1399,1212,1211,10092,71$ & $3.84 \times 10^{-7}$ \\
hsa01100 & Metabolic pathways & $3945,6888,7381,7298,521,522,4124,81490,2597$, & $4.82 \times 10^{-7}$ \\
hsa05016 & Huntington's disease & $4719,5033,2744,6241,3155,283871,5106,55256$ & $5.06 \times 10^{-7}$ \\
hsa03010 & Ribosome & $7381,1212,1211,522,4719,160,293$ & $6.59 \times 10^{-7}$ \\
hsa05130 & Pathogenic Escherichia coli infection & $4691,10971,10092,71$ & $1.62 \times 10^{-6}$ \\
hsa04666 & Fc gamma R-mediated phagocytosis & $1399,4082,65108,10092$ & $2.17 \times 10^{-5}$ \\
hsa04961 & Endocrine and other factor-regulated & $1212,1211,160$ & \\
& calcium reabsorption & $6233,51116,6156,6138,6218,6224$ & \\
\hline
\end{tabular}

KEGG, Kyoto Encyclopedia of Genes and Genomes; HUVECs, human umbilical vein endothelial cells.

As shown in Table 3, pathways such as protein export (hsa03060), Shigellosis (hsa05131), aminoacyl-tRNA biosynthesis (hsa00970), bacterial invasion of epithelial cells (hsa05100), metabolic pathways (hsa01100), and Huntington's disease (hsa05016) were the most significantly enriched KEGG pathways associated with hypoxanthineinduced oxidative stress in HUVECs.

\section{PPI network analysis of the DEPs}

In the animal body, when cells are stimulated by internal and external oxidative factors, the production of ROS is increased, disrupting the balance between the oxidation and anti-oxidation systems, leading to oxidative stress (3-5). Excessive ROS accumulation can activate factors such as nuclear factor E2-related factor 2(Nrf2), nuclear factor- $\kappa \mathrm{B}$ (NF- $\mathrm{KB}$ ), and mitogen-activated protein kinase (MAPK) to regulate the expression of oxidant and antioxidant factors $(1,2)$. Thus, in this study, we selected 20 DEPs involved in oxidative stress, cell apoptosis, and DNA damage (XRCC6, LDHB, TALDO1, RPS27A, UQCRB, GLRX3, APEX1, PPP1R10, SUB1, TOP2A, RIF1, GAPDH, NONO, SLC25A6, CPD, HMOX1, NUCB1, DEK, CHTOP, and ING2) through literature research, and then identified sixteen impotence-related proteins among them, namely NOS1, PRL, PDE5A, KLK3, VIP, SHBG, NOS3, KCNMA1, NOS2, EDN1, PDE3A, CYP3A4, ALDH7A1, KNG1, SRD5A1, ARG2, and SEPT3 using the MalaCards database (http://www.malacards.org/). These proteins were imported into STRING and further analyzed by CytoScape (Figure 5).

\section{Role of HMOX1 in bypoxanthine-induced oxidative stress in HUVECs}

To verify the results of iTRAQ-MS, we selected the significant DEPs NCL, XRCC6, UQCRB, NONO, HMOX1, NUCB1, DEK, and CHTOP for RT-qPCR verification. As shown in Figure 6A, compared to those in the normal control group, the mRNA levels of NCL and NUCB1 were significantly upregulated, while that of HMOX1 was downregulated in hypoxanthine-treated cells. Thus, our real-time PCR results corroborated the iTRAQMS results.

The iTRAQ results showed that the expression of HMOX1 was downregulated in oxidative stress-exposed HUVECs, indicating that this gene may play an important role in the oxidative stress response in HUVECs. To test this hypothesis, we constructed an HMOX1 overexpression plasmid (pcDNA-HMOX1) and its control plasmid (pcDNA-Con). Cells treated with hypoxanthine were transfected with the two plasmids. As indicated in Figure 6B, the transfection did not alter cell viability compared with that of the normal control. As shown by Western blotting, the expression of HMOX1 was downregulated in hypoxanthine-treated HUVECs, while transfection with the 

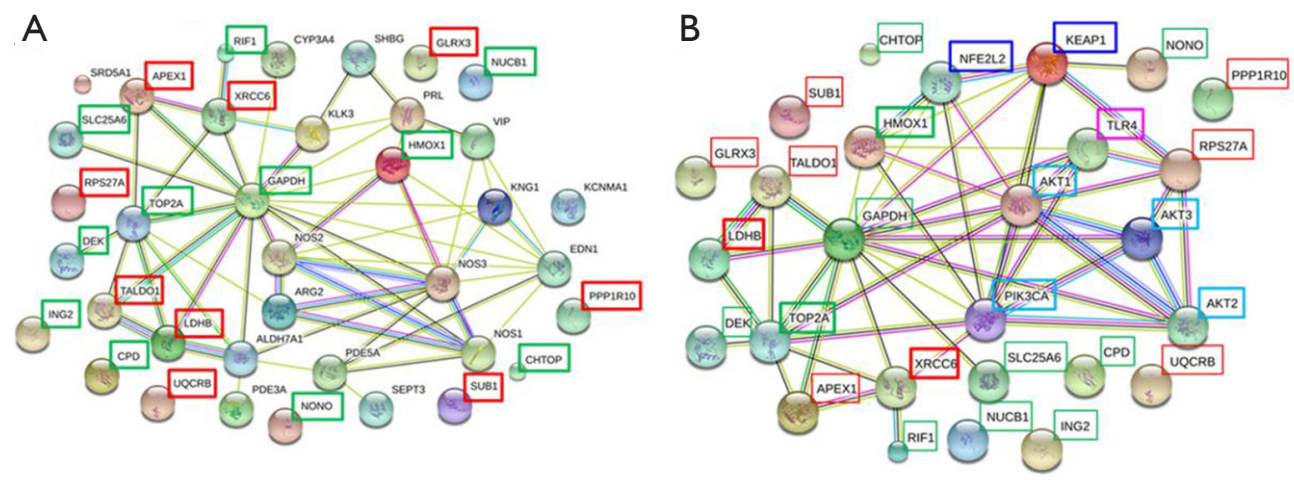

Figure 5 Protein-protein interaction network analysis. (A) Network of twenty differentially expressed proteins (DEPs) involved in the oxidative stress response, cell apoptosis, and DNA damage, as well as erectile dysfunction-related proteins; (B) network of twenty DEPs associated with the Keap1-Nrf2-ARE, PI3K/AKT, and toll-like receptor (TLR) signaling pathways. Red and green boxes indicate upregulated and downregulated proteins, respectively. Blue, light blue, and pink boxes indicate proteins associated with the Keap1-Nrf2ARE, PI3K/AKT, and TLR signaling pathways, respectively.

overexpression plasmid had the opposite effect (Figure 6C). SOD, catalase (CAT), and NO levels were decreased upon hypoxanthine treatment, and could be restored by HMOX1 overexpression (Figure 6D,E,F). HMOX1 is known to be a downstream target of AKT (20). We activated the AKT pathway using insulin-like growth factor 1 (IGF-1), and this stimulation increased the expression of HMOX1 (Figure $6 G$ ) and increased NO production (Figure 6H). These data suggest that HMOX1 is important in the hypoxanthineinduced oxidative stress response of HUVECs.

\section{Discussion}

ED has become a worldwide issue affecting the health of men; its incidence has risen sharply not only in Europe and North America, but also in other countries (21). Penile erection is a vascular phenomenon, and blood flow plays a central role in the erectile mechanism $(22,23)$. Hyperglycemia affects the formation of capillary basement membranes and macromolecular polysaccharides, and it induces endothelial cell membrane glycation $(24,25)$. Vascular endothelial injury is a key factor in the occurrence and development of vascular diseases in diabetic patients (26). There is known to be a close relationship between vascular disease and diabetic ED (2). Diabetic patients with macrovascular diseases are vulnerable to internal iliac artery and sponge spiral atherosclerosis, which can lead to reduced blood pressure, blood perfusion to the cavernous sinus, and penile erection hardness (27). Furthermore, capillary microcirculation in diabetic patients with organic impotence shows significant pathological changes, including poor microvascular filling and a significant reduction in the number of blood vessels $(27,28)$.

Corpus cavernosum endothelial cells, endothelial cells arranged on the inner surface of the cavernous sinus, are one of the basic components of penile vessels; their major function is the synthesis of vasodilatory factors and contractile factors (such as $\mathrm{NO}$ and endothelin), which play important roles in penile erection. However, the molecular mechanisms involved in the oxidative stress response of endothelial cells have not been fully determined. In particular, no studies have yet performed a differential proteome analysis of endothelial cells in response to oxidative stress. Thus, in the current study, we utilized iTRAQ to identify DEPs and further explore the pathologic mechanisms of the oxidative stress response in HUVECs. In total, we identified 66 upregulated and 76 downregulated proteins; these proteins are thought to play a role in the oxidative stress response in HUVECs. Among them, we verified the mRNA expression of several dysregulated proteins using RT-qPCR. Further, using gene overexpression and knockdown, we found that HMOX1 was critical for the oxidative stress response in HUVECs.

HMOX 1 is critical in the defense response against oxidant-induced injury in many pathological conditions (29-31). HMOX1 is known to be regulated by oxidative stress-promoting stimuli including hypoxia, hyperoxia, heat shock, excess heme accumulation, NO, and endotoxins $(32,33)$. HMOX1 shows anti-inflammatory, antioxidant, antiapoptotic and antiproliferative effects $(34,35)$, and is 

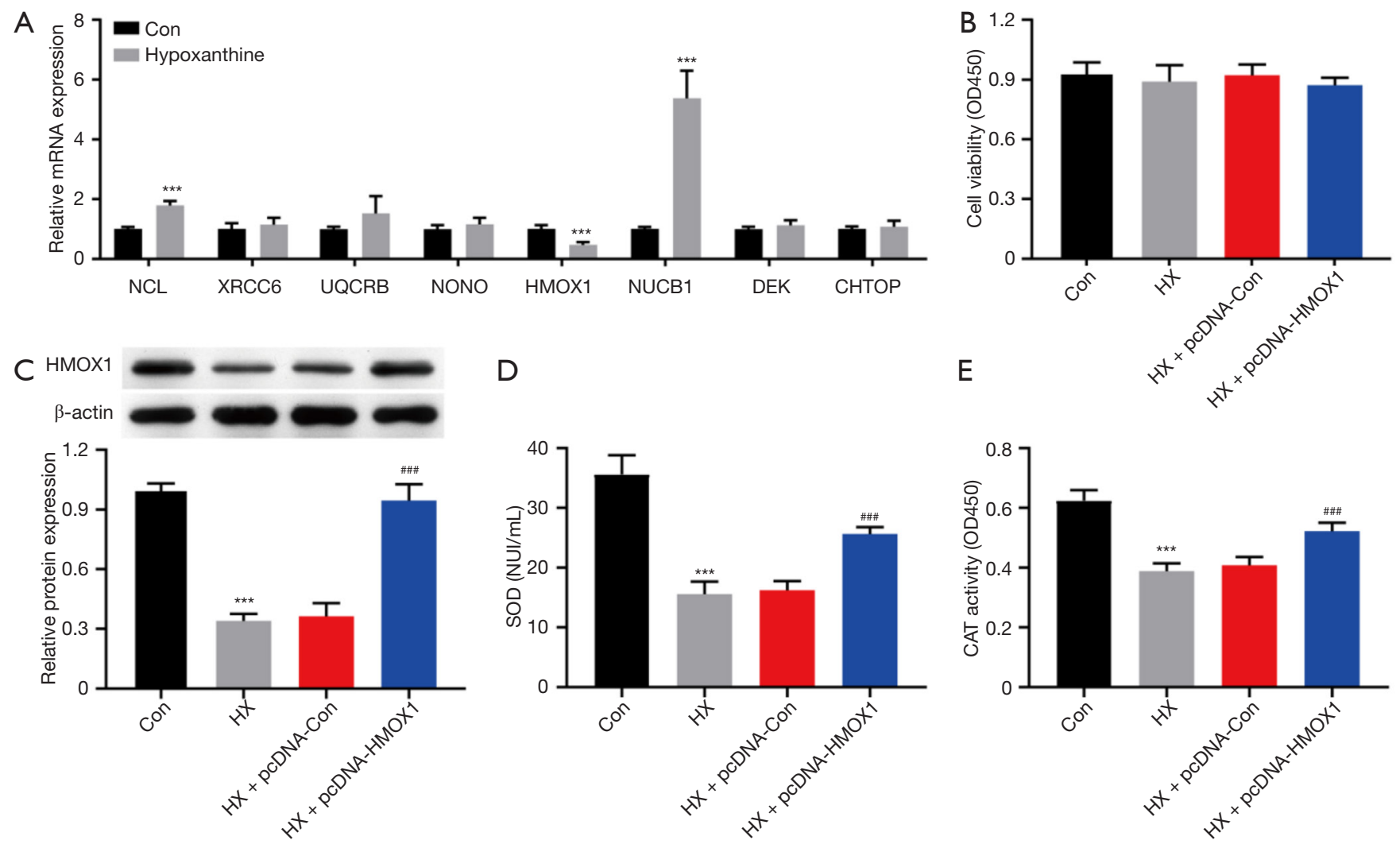

$\mathrm{F}$

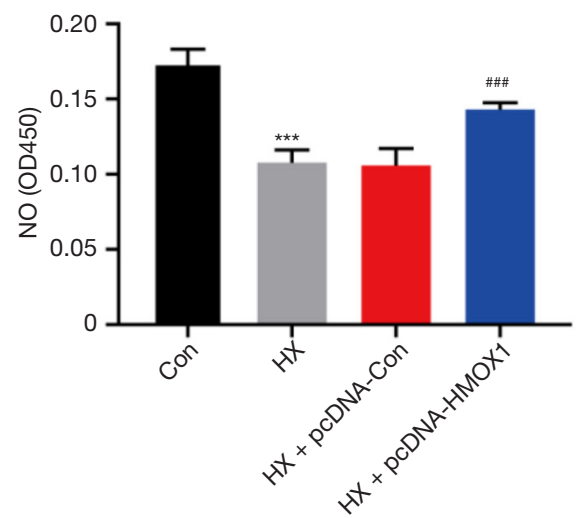

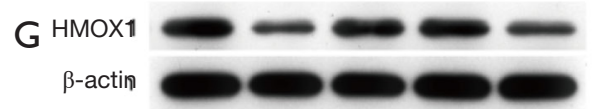

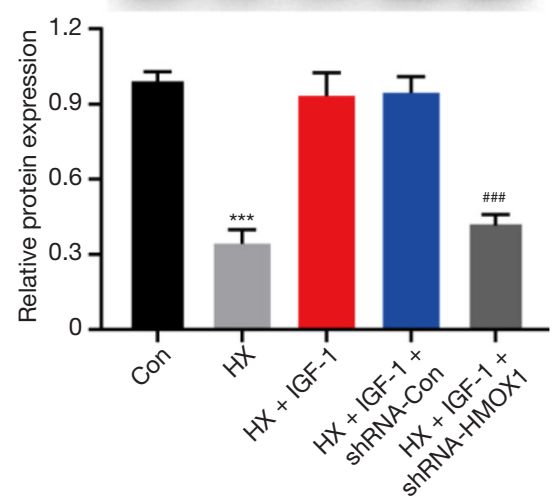

$\mathrm{H}$

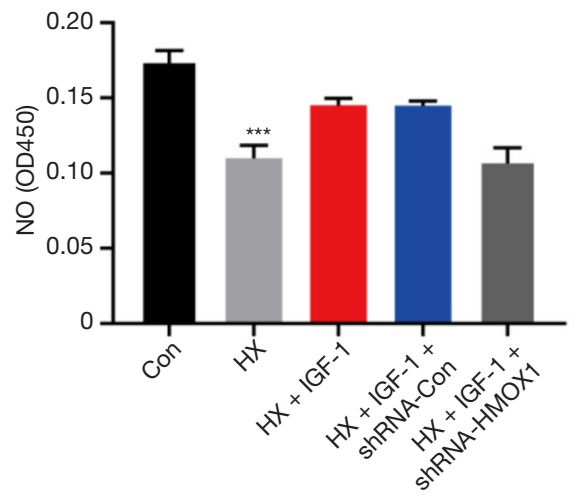

Figure 6 Role of heme oxygenase 1 (HMOX1) in the hypoxanthine-induced oxidative stress response in human umbilical vein endothelial cells (HUVECs). (A) Cultured HUVECs were treated with 1,000 ng/mL hypoxanthine or left untreated (control, Con), and the cells were subjected to RT-qPCR to verify the results of iTRAQ. The relative mRNA expression levels of the indicated genes are shown. ${ }^{* * *}, \mathrm{P}<0.001$ versus the control group. (B) HUVECs were treated with (HX) or without (Con) hypoxanthine, or together with HMOX1 control (HX + pcDNA-Con) or HMOX1 overexpression plasmids (HX + pcDNA-HMOX1); thereafter, cell viability was determined in each group. (C) Western blot validation of the plasmid transfection, with $\beta$-actin as a loading control. (D,E,F) Levels of superoxide dismutase (SOD) (D), catalase (CAT) (E), and NO (F) in each group. (G) Cultured HUVECs were treated with or without hypoxanthine together with the AKTpathway activator IGF-1 or HMOX1 knockdown plasmids. Western blot validation and quantification of the plasmid transfection, with $\beta$-actin

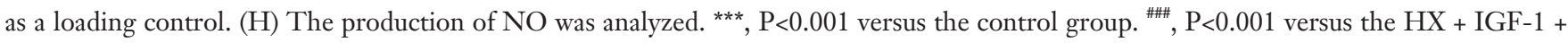
shRNA-Con group. 
thus considered a cytoprotective enzyme (36). HMOX1 was previously found to modulate oxidative stress and inflammation and regulate cell cycle progression to prevent apoptosis in acute kidney injury (37). NO stimulates HMOX1 expression through the Nrf2/antioxidant responsive element (ARE) complex and promotes the survival of vascular smooth muscle cells (38). Additionally, we found that hypoxanthine treatment downregulated HMOX1 in HUVECs, indicating that HMOX1 regulates a variety of processes related to penile function in vascular smooth muscle cells and endothelial cells. Furthermore, HMOX1 is known to attenuate lipogenesis disorders, as its upregulation reduces visceral and subcutaneous fat accumulation and improves insulin sensitivity $(39,40)$; thus, HMOX1 plays a major role in DED. HMOX1 has been reported to be the downstream target of AKT (20); in the current study, we demonstrated that AKT activity affected HMOX1 expression. Furthermore, reduced phosphoinositide 3-kinase (PI3K)/AKT/endothelial nitric oxide synthase (eNOS) activity was previously shown to be closely related to metabolic syndrome in a rat model of ED (41). Our data showed that activation of the AKT pathway increased NO production, consistent with previous reports $(41,42)$.

Although the pathogenesis of DED is clear, the intracellular mechanism of the injury of penile endothelial cells is not. In this experiment, we used proteomics to analyze the difference in protein expression in endothelial cell injury induced by high glucose, which provided a basis for understanding the occurrence, development, and prognosis of DED. According to the function and location of these proteins, we can further understand the mechanism of dynamic changes in cells when DED occurs. These DEPs are mainly related to oxidative stress and energy metabolism, suggesting that the high glucose environment of penile epithelial cells in diabetic patients is closely related to inflammatory responses. It is confirmed that the damage of high glucose load can be regulated by reducing oxidative stress damage and affecting energy metabolism. The limitation of this experiment is that the functions of other molecules beyond that of HMOX1 have yet to be tested, and these are expected to represent other potential markers of DED. Although our in vitro study advances understanding of the role of HMOX1 in DED, it is necessary to confirm our findings in multiple cell lines and in vivo experiments in the future.

\section{Conclusions}

The present study provides the first overview of protein alterations in HUVECs under oxidative stress. Our identification of DEPs by iTRAQ analysis revealed a comprehensive interaction network in HUVECs during their oxidative stress response. Several significantly dysregulated proteins were identified to be associated with the pathological response of VECs under ED. Further functional exploration should be implemented to reveal pathologic mechanisms and identify new therapeutic targets for preventing ED.

\section{Acknowledgments}

Funding: This work was supported by the National Natural Science Foundation of China (grant No. 81871158 , 81370705 and 81571432).

\section{Footnote}

Conflicts of Interest: All authors have completed the ICMJE uniform disclosure form (available at http://dx.doi. org/10.21037/tau.2020.03.11). The authors have no conflicts of interest to declare.

Ethical Statement: The authors are accountable for all aspects of the work in ensuring that questions related to the accuracy or integrity of any part of the work are appropriately investigated and resolved.

Open Access Statement: This is an Open Access article distributed in accordance with the Creative Commons Attribution-NonCommercial-NoDerivs 4.0 International License (CC BY-NC-ND 4.0), which permits the noncommercial replication and distribution of the article with the strict proviso that no changes or edits are made and the original work is properly cited (including links to both the formal publication through the relevant DOI and the license). See: https://creativecommons.org/licenses/by-nc$\mathrm{nd} / 4.0 \%$.

\section{References}

1. Shaw JE, Sicree RA, Zimmet PZ. Global estimates of the prevalence of diabetes for 2010 and 2030. Diabetes Res Clin Pract 2010;87:4-14. 
2. Castela Â, Costa C. Molecular mechanisms associated with diabetic endothelial-erectile dysfunction. Nat Rev Urol 2016;13:266-74.

3. Shamloul R, Ghanem H. Erectile dysfunction. Lancet 2013;381:153-65.

4. McMahon CG. Erectile dysfunction. Intern Med J 2014;44:18-26.

5. Lizza EF, Rosen RC. Definition and classification of erectile dysfunction: report of the Nomenclature Committee of the International Society of Impotence Research. Int J Impot Res 1999;11:141-3.

6. Malavige LS, Levy JC. Erectile dysfunction in diabetes mellitus. J Sex Med 2009;6:1232-47.

7. Tarbell JM, Simon SI, Curry FR. Mechanosensing at the vascular interface. Annu Rev Biomed Eng 2014;16:505-32.

8. Newman HF, Tchertkoff V. Penile vascular cushions and erection. Invest Urol. 1980;18:43-5.

9. Saenz de Tejada I, Goldstein I, Krane RJ. Local control of penile erection. Nerves, smooth muscle, and endothelium. Urol Clin North Am 1988;15:9-15.

10. Castela A, Gomes P, Silvestre R, et al. Vasculogenesis and diabetic erectile dysfunction: how relevant is glycemic control? J Cell Biochem 2017;118:82-91.

11. Brownlee M. Biochemistry and molecular cell biology of diabetic complications. Nature 2001; 414:813-20.

12. Nowotny K, Jung T, Höhn A, et al. Advanced glycation end products and oxidative stress in type 2 diabetes mellitus. Biomolecules 2015;5:194-222.

13. Afanas'ev I. Signaling of reactive oxygen and nitrogen species in Diabetes mellitus. Oxid Med Cell Longev 2010;3:361-73.

14. Newsholme P, Haber EP, Hirabara SM, et al. Diabetes associated cell stress and dysfunction: role of mitochondrial and non-mitochondrial ROS production and activity. J Physiol 2007;583:9-24.

15. Musicki B, Kramer MF, Becker RE, et al. Inactivation of phosphorylated endothelial nitric oxide synthase (Ser-1177) by O-GlcNAc in diabetes-associated erectile dysfunction. Proc Natl Acad Sci USA 2005;102:11870-5.

16. Ryu JK, Kim DJ, Lee T, et al. The role of free radical in the pathogenesis of impotence in streptozotocin-induced diabetic rats. Yonsei Med J 2003;44:236-41.

17. Kim YJ, Ryu HM, Choi JY, et al. Hypoxanthine causes endothelial dysfunction through oxidative stressinduced apoptosis. Biochem Biophys Res Commun. 2017;482:821-7.

18. Grishko VI, Driggers WJ, LeDoux SP, et al. Repair of oxidative damage in nuclear DNA sequences with different transcriptional activities. Mutat Res 1997;384:73-80.

19. Qi DH, Luo GM, Zhou L, et al. Protection of myocardial mitochondria against oxidative damage by seleniumcontaining abzyme m4G3. Appl Biochem Biotechnol 1999; 82:167-73.

20. Salinas M, Wang J, Rosa de Sagarra M, et al. Protein kinase $\mathrm{Akt} / \mathrm{PKB}$ phosphorylates heme oxygenase-1 in vitro and in vivo. FEBS Lett 2004;578:90-4.

21. Hatzimouratidis K, Amar E, Eardley I, et al. Guidelines on male sexual dysfunction: erectile dysfunction and premature ejaculation. Eur Urol 2010;57:804-14.

22. La Favor JD, Anderson EJ, Hickner RC, et al. Erectile dysfunction precedes coronary artery endothelial dysfunction in rats fed a high-fat, high-sucrose, Western pattern diet. J Sex Med 2013;10:694-703.

23. Chai SJ, Barrett-Connor E, Gamst A. Small-vessel lower extremity arterial disease and erectile dysfunction: The Rancho Bernardo study. Atherosclerosis 2009;203:620-5.

24. Aronson D. Hyperglycemia and the pathobiology of diabetic complications. In: Fisman EZ, Tenenbaum A. editors. Cardiovascular diabetology: clinical, metabolic and inflammatory facets. Adv Cardiol. Volume 45. Basel: Karger, 2008:1-16.

25. Xu Y, He Z, King GL. Introduction of hyperglycemia and dyslipidemia in the pathogenesis of diabetic vascular complications. Curr Diab Rep 2005;5:91-7.

26. Vinik AI, Erbas T, Park TS, et al. Platelet dysfunction in type 2 diabetes. Diabetes Care 2001;24:1476-85.

27. Maiorino MI, Bellastella G, Esposito K. Diabetes and sexual dysfunction: current perspectives. Diabetes Metab Syndr Obes 2014;7:95-105.

28. Turek SJ, Hastings SM, Sun JK, et al. Sexual dysfunction as a marker of cardiovascular disease in males with 50 or more years of type 1 diabetes. Diabetes Care 2013;36:3222-6.

29. Lenoir O, Gaillard F, Lazareth H, et al. Hmox1 deficiency sensitizes mice to peroxynitrite formation and diabetic glomerular microvascular injuries. J Diabetes Res 2017;2017:9603924.

30. Mustafa S, Weltermann A, Fritsche R, et al. Genetic variation in heme oxygenase 1 (HMOX1) and the risk of recurrent venous thromboembolism. J Vasc Surg 2008;47:566-70.

31. Waza AA, Hamid Z, Ali S, et al. A review on heme oxygenase-1 induction: is it a necessary evil. Inflamm Res 2018;67:579-88.

32. Ghosh D, Ulasov IV, Chen L, et al. TGF $\beta$-responsive HMOX1 expression is associated with stemness and 
invasion in glioblastoma multiforme. Stem Cells 2016;34:2276-89.

33. Moreno-Navarrete JM, Ortega F, Rodríguez A, et al. HMOX1 as a marker of iron excess-induced adipose tissue dysfunction, affecting glucose uptake and respiratory capacity in human adipocytes. Diabetologia 2017;60:915-26.

34. Hou W, Tian Q, Zheng J, et al. MicroRNA-196 represses Bach1 protein and hepatitis $\mathrm{C}$ virus gene expression in human hepatoma cells expressing hepatitis $\mathrm{C}$ viral proteins. Hepatology 2010;51:1494-504.

35. Zhan CY, Chen D, Luo JL, et al. Protective role of downregulated microRNA-31 on intestinal barrier dysfunction through inhibition of NF- $\kappa \mathrm{B} / \mathrm{HIF}-1 \alpha$ pathway by binding to HMOX1 in rats with sepsis. Mol Med 2018;24:55.

36. Mahawar L, Shekhawat GS. Haem oxygenase: A functionally diverse enzyme of photosynthetic organisms and its role in phytochrome chromophore biosynthesis, cellular signalling and defence mechanisms. Plant Cell Environ 2018;41:483-500.

37. Bolisetty S, Zarjou A, Agarwal A. Heme oxygenase 1 as a therapeutic target in acute kidney injury. Am J Kidney Dis
2017;69:531-45.

38. Liu XM, Peyton KJ, Ensenat D, et al. Nitric oxide stimulates heme oxygenase-1 gene transcription via the Nrf2/ARE complex to promote vascular smooth muscle cell survival. Cardiovasc Res 2007;75:381-9.

39. Burgess A, Li M, Vanella L, et al. Adipocyte heme oxygenase-1 induction attenuates metabolic syndrome in both male and female obese mice. Hypertension 2010;56:1124-30.

40. Li M, Kim DH, Tsenovoy PL, et al. Treatment of obese diabetic mice with a heme oxygenase inducer reduces visceral and subcutaneous adiposity, increases adiponectin levels, and improves insulin sensitivity and glucose tolerance. Diabetes 2008;57:1526-35.

41. Li R, Cui K, Liu K, et al. Metabolic syndrome in rats is associated with erectile dysfunction by impairing PI3K/ Akt/eNOS activity. Sci Rep 2017;7:13464.

42. Zhang X, Zhao F, Zhao JF, et al. PDGF-mediated PI3K/ AKT/beta-catenin signaling regulates gap junctions in corpus cavernosum smooth muscle cells. Exp Cell Res 2018;362:252-9.
Cite this article as: Zhu P, Qi T, Huang ZS, Li H, Wang B, Feng JX, Ma S, Xiao HJ, Tang YX, Liu W, Chen J. Proteomic analysis of oxidative stress response in human umbilical vein endothelial cells (HUVECs): role of heme oxygenase 1 (HMOX1) in hypoxanthine-induced oxidative stress in HUVECs. Transl Androl Urol 2020;9(2):218-231. doi: 10.21037/ tau.2020.03.11 


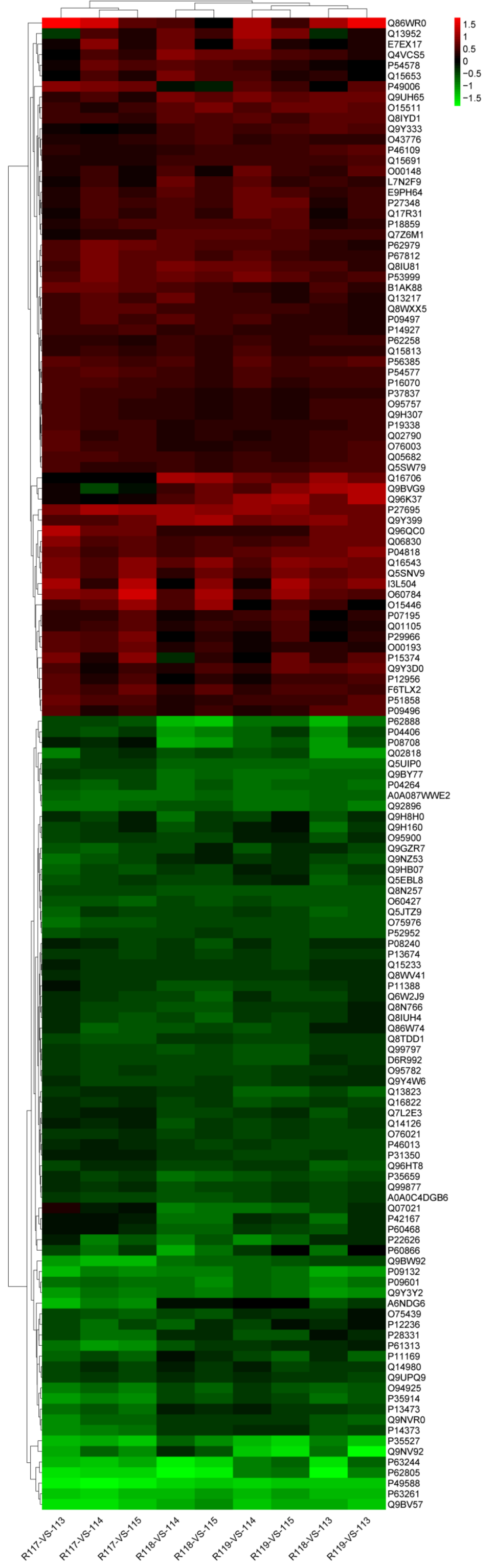

Figure S1 Heatmap Analysis of differentially expressed proteins (DEPs) between hypoxanthine-treated and untreated human umbilical vein endothelial cells (HUVECs). 